\title{
A METHOD FOR COMPOSITE FAULT DETECTION AND ISOLATION USING OVERLAPPING DECOMPOSITION
}

\author{
Rogério Bastos Quirino* \\ rquirino@certto.com.br md.cefetpr.br
}

\author{
Celso Pascoli Bottura ${ }^{\dagger}$ \\ bottura@dmcsi.fee.unicamp.br
}

\footnotetext{
*Departamento de Tecnologia Eletromecânica, CEFET-PR. 85.884-000 Medianeira, PR, BRAZIL

†Laboratório de Controle e Sistemas Inteligentes, Departamento de Máquinas, Componentes e Sistemas

Inteligentes, UNICAMP. P.O. Box 6101, 13083-970 Campinas, SP, BRAZIL
}

\begin{abstract}
In this article, a method is developed for fault detection in linear, stochastic, interconnected dynamic systems, based on designing a set of partially decentralized Kalman filters for the subsystems resulting from the overlapping decomposition of the overall large scale system. The faulty sensors can be detected and isolated by comparing the estimated values of a single state from partially decoupled Kalman filters. The method is applied to an example system with two sensors.
\end{abstract}

KEYWORDS: fault detection, overlapping, Kalman filters, sensor failures, sensors.

\section{RESUMO}

Neste artigo é desenvolvido um método de detecção de falhas em sistêmas dinâmicos acoplados lineares e estocásticos, baseado no projeto de filtros de Kalman parcialmente descentralizados aplicados aos subsistemas resultantes da decomposição "overlapping"do sistema global. A detecção da(s) falha(s) e o isolamento do(s) sensor(es) falho(s) são feitos através da comparação dos valores estimados dos estados redundantes dos filtros de Kalman parcialmente desacoplados. Um modelo de aplicação com dois sensores é utilizado na validação do mé-

Artigo submetido em $20 / 12 / 2000$

1a. Revisão em 3/6/2002; 2a Revisão em 11/2/2003

Aceito sob recomendação do Ed. Assoc. Prof. Liu Hsu todo.

PALAVRAS-CHAVE: Detecção de falhas, decomposição com sobreposição, filtros de Kalman, falhas de sensores, sensores.

\section{INTRODUCTION}

An important problem facing engineers in designing complex industrial processes, which has attracted scientists and researchers in the field of systems science, is the problem of failure detection in running systems (Willsky, 1976 ; Isermman, 1984 ; Frank, 1990).

The failure detection problem is an extremely complex one, and the choice of an appropriate design depends heavily on the particular application.

An important issue to be considered by the designer of failure detection systems is the issue of computational complexity. One clearly needs a scheme that has reasonable time requirements. It would also be useful to have a design methodology that admits a range of implementations, allowing a trade-off study between system complexity and performance.

In addition, it would be desirable to have a design that takes advantage of computer capabilities and structures, e.g., designs that are amenable to parallel and distributed implementations where the environment under consideration is a multisensor network. 


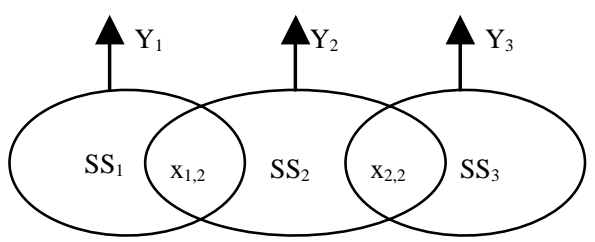

Figure 1: Overlapping decomposition for three subsystems.

We base this paper on the overlapping decomposition technique (Ikeda and Siljak, 1980) combined with a parallel and distributed Kalman filter proposed in Quirino and Bottura, (2001) in order to yield a sensors multiple fault detection and isolation method.

A partially decoupled estimation methodology requires, when constructed, communication between the local filters. In principle, such communication is in opposition to the decentralization philosophy of the overlapping technique. However, through this communication we achieve simultaneously two important aims: 1) The construction of a consistent estimator that complies with the detection structure; 2) The improvement of the performance of the detection system regarding its capacity to detect and isolate single faults as well as multiple faults.

The apparent contradiction that arises using distributed estimation techniques, developed in the two last decades and discussed in Quirino and Bottura (2001), jointly with the overlapping decomposition technique (Krtolica and Siljak, 1980), can have hindered progress in the development of methods for monitoring, distributed sensor fault detection and isolation.

Sensor Fault Detection (SFD) techniques for large-scale systems have been developed (Singh et al.,1983; Benkherouf and Allidina, 1987; Hassan et al., 1992), using an overlapping decomposition method.

This concept is accomplished (Ikeda and Siljak, 1980; Ikeda et al., 1981) by expanding the original system into a larger system comprised of a collection of interconnected subsystems. Although the order of the expanded system is higher than that of the original system due to the introduction of overlapping, the order of each subsystem (and consequently, the order of each one of the decentralized state estimators) is much lower. Furthermore, it is only required that the subsystems be locally observable. This is easier to test for than the observability of the original system.

In the method described by Hassan et al. (1992) state observers for the interconnected subsystems are designed independently, neglecting the interaction terms between the subsystems.

The results obtained for suboptimal decentralized control can be applied to the problem of decentralized state estimation (Krtolica and Siljak, 1980), by using duality.

It is thus possible to detect and identify a faulty sensor by comparing the discrepancies between estimates of the same (overlapping) state provided by different sub-observers. However, the sensor failures would only be detected and isolated correctly if they were assumed to occur one at a time.

In the following, we illustrate how a global system partitioned into three subsystems with an expanded state space model in such a way as to generate two overlapped states.

Consider the case of overlapping decomposition for three partitioned subsystems, with two overlapped states $\mathrm{x}_{1,2}$ and $\mathrm{x}_{2,2}$, as shown in Figure 1.

With respect to Figure 1, the rows of Table 1 show us each one of the ambiguous situations (column 1) between simple (column 2) and multiple (column 3) faults, and the criterions (column 4) to them related. Such criterions (column 4) were established in Hassan et al. (1992) in order to treat uniquely simple fault (column 2) occurrences.

In this article we propose alternative criterions to those established in Hassan et al. (1992), in order to avoid the ambiguity situations as shown in Table 1 and diagnose correctly simple faults as well as composite faults.

The use of the method developed by Hassan et al. (1992) would generate ambiguous situations and as consequences missed detections and false alarms. For example, in situation 4, as shown in Table 1, the composite failure of sensors $y_{1}$ and $y_{2}$, would produce a false alarm in sensor $y_{3}$ and missed detections in sensors $y_{1}$ and $y_{2}$.

In situation 1, as shown in Table 1, composite failure in all three sensors would not be diagnosed.

Thus, the SFD scheme proposed in Hassan et al. (1992) would fail to detect composite faults.

An extension of this method, which would permit identification and isolation of a single malfunctioning sensor as well as of sensors simultaneously or sequentially faulty , is the proposal of this work. The proposal is based on the approach for distributed Kalman filtering (Quirino and Bottura, 2001), developed from an hierarchical es- 
Table 1: Ambiguous situations for single and composite faults

\begin{tabular}{|c|c|c|c|}
\hline Sit. & Single Faults & Composite Faults & $\begin{array}{l}\text { Module of Estimation } \\
\text { Discrepances }\end{array}$ \\
\hline 1 & no fault & faults in $y_{1}, y_{2}$ and $y_{3}$ & $\left|Z_{1,2}\right|<\varepsilon_{1}$ and $\left|Z_{2,2}\right|<\varepsilon_{2}$ \\
\hline 2 & fault in $y_{1}$ & faults in $y_{2}$ and $y_{3}$ & $\left|Z_{1,2}\right|>\varepsilon_{1}$ and $\left|Z_{2,2}\right|<\varepsilon_{2}$ \\
\hline 3 & fault in $y_{2}$ & faults in $y_{1}$ and $y_{3}$ & $\left|Z_{1,2}\right| \varepsilon_{1}$ and $\left|Z_{2,2}\right|>\varepsilon_{2}$ \\
\hline 4 & fault in $y_{3}$ & faults in $y_{1}$ and $y_{2}$ & $\left|Z_{1,2}\right|<\varepsilon_{1}$ and $\left|Z_{2,2}\right|>\varepsilon_{2}$ \\
\hline \multicolumn{4}{|c|}{$\begin{array}{l}Z_{1,2}=\left(\chi_{1,2}\right)_{S S 1^{-}}\left(\chi_{1,2}\right)_{S S 2} ; \\
Z_{2,2}=\left(\chi_{2,2}\right)_{S S 2^{-}}\left(\chi_{2,2}\right)_{S S 3} ; \\
\varepsilon_{1}, \varepsilon_{2} \equiv \text { constants to be determined. } \\
\left(\chi_{1,2}\right)_{S S i} \equiv \text { estimate of the overlapped state } x_{1,2} \text { by the totally } \\
\text { decoupled Kalman filter of } i \text {-th subsystem. } \\
\left(\chi_{2,2}\right)_{S S j} \equiv \text { estimate of the overlapped state } x_{2,2} \text { by the totally } \\
\text { decoupled Kalman filter of } j \text {-th subsystem. } i=1,2 ; j=2,3 \text {. }\end{array}$} \\
\hline
\end{tabular}

timation structure. It is optimal in the sense of Kalman filtering and is based on the multiple projections (successive orthogonalizations) method (Quirino et al., 1998).

The extension involves the use of a duality that exists between two state space representations.

It is derived from the application of an approach using the coupling and noise terms of the original system.

The algebraic structure developed is suboptimal, due to the fact that it does not take into account the updating of the state prediction based on the multiple innovations.

The article is organized as follows. Section two is concerned with the expansion and decomposition of a dynamical system into a set of overlapping subsystems. The SFD procedure is described in section 3 and simulations illustrating the method are given in section 4 . Finally, some conclusions are given in section 5 .

\section{SYSTEM MODEL AND OVERLAP- PING DECOMPOSITION}

Consider a large-scale linear interconnected system S, which is described by the following state and output equations:

$$
\begin{array}{cl}
S: & x_{k+1}=A x_{k}+w_{k} \\
& y_{k}=H_{k} x_{k}+v_{k}
\end{array}
$$

where $x \in R^{n}, w_{k} \in R^{n}$ is the state noise vector, $y_{k} \in$ $R^{m}$ is the output measurement vector and $v_{k} \in R^{m}$ is the noise disturbing the output. $A$ and $H$ are the system matrices of appropriate dimensions, in which $H$ is assumed to be a block-diagonal matrix with $N$ blocks corresponding to $N$ subsystems.

For the above system given by eqns. 1 and 2, we have the following assumptions:

(1) $w_{k}$ and $v_{k}$ are Gaussian random vectors with zero mean and covariances respectively given by $E\left\{w_{j} w_{k}^{t}\right\}=$ $Q \delta_{j k}, E\left\{v_{j} v_{k}^{t}\right\}=R \delta_{j k}$

(2) The disturbance vectors are uncorrelated, i.e., $E\left\{v_{j} w_{k}^{t}\right\}=0 \quad \forall j, k$.

(3) The initial state vector $x(0)$ is a Gaussian random vector with mean $E\{x(0)\}=X_{0}$ and covariance $E\left\{\left[x(0)-X_{0}\right]\left[x(0)-X_{0}\right]^{t}\right\}=P_{0}$.

(4) $x(0)$ and the noise vectors $v_{k}$ and $w_{k}$ are uncorrelated, i.e., $E\left\{x(0) v_{k}^{t}\right\}=0, E\left\{x(0) w_{k}^{t}\right\}=0 \quad \forall k$.

The system $S$ described by equations (1) and (2) can be expanded into another system $\underline{\underline{S}}$ using a linear transformation

$$
\underline{\underline{x}}_{k}=T x_{k}
$$

where $\underline{\underline{x}} \in R \underline{\underline{n}}(\underline{\underline{n}}>\mathrm{n})$ and $T$ is a $\underline{\underline{n}} \times \mathrm{n}$ constant transformation matrix. The expanded system is given by:

$$
\begin{gathered}
\underline{\underline{S}}: \quad \underline{\underline{x}}_{k+1}=\underline{\underline{A x}} k+\underline{\underline{w}}_{k} \\
y_{k}=\underline{\underline{H \underline{x}}} k+v_{k}
\end{gathered}
$$

where $\underline{\underline{w}}_{k}$ is the expanded state noise and $\underline{\underline{A}}$ and $\underline{\underline{H}}$ are the new system matrices (with dimensions $\underline{\underline{\underline{n}}} \times \underline{\underline{n}}, \underline{\bar{m}} \times \underline{\underline{n}}$ respectively) given by:

$$
\begin{gathered}
\underline{\underline{A}}=T A T^{I}+M ; \underline{\underline{H}}=H T^{I}+L \\
T^{I}=\left(T^{t} T\right)^{-1} T^{t}
\end{gathered}
$$

where $M$ and $L$ are complementary matrices of appropriate dimensions (Ikeda and Siljak, 1980).

$T^{I}$ is the generalized inverse of $T \in R^{n x n}$, which is a 
transformation matrix given by:

$$
T=\left[\begin{array}{ccccccc}
I_{1,1} & & & & & & \\
& I_{1,2} & & & & & \\
& I_{1,2} & & & & & \\
& & I_{2,1} & & & & \\
& & & \ddots & & & \\
& & & & I_{N-1,1} & & \\
& & & & & I_{N-2,2} & \\
& & & & & & I_{N-2,2} \\
& & & & & & I_{N, N}
\end{array}\right]
$$

where $I_{i, 1}$ is an identity matrix with dimension $\left(n_{i}-n_{i, 2}\right)$ $\times\left(n_{i}-n_{i, 2}\right) ; I_{i, 2}$ is an identity matrix with dimension $n_{i, 2} \times n_{i, 2}, \mathrm{i}=1, \ldots, \mathrm{N}$.

\section{FAULT DETECTION METHOD}

In this section, we consider the problem of detecting the malfunctioning sensors of the augmented system $\underline{S}$, which comprises $N$ overlapping subsystems. This will be achieved through the design of Partially Decentralized Hierarchical Kalman Filters (PDHKF), presented in Quirino and Bottura, (2001) for the subsystems and by comparing the estimated states, which are obtained by two successive filters for each subsystem.

The $i$ th subsystem $\underline{\underline{S}}$ derived from the expansion is described by the following equations:

$$
\begin{gathered}
\underline{S}_{i}: \underline{x}_{k+1}^{i}=\underline{\underline{A}}_{k}^{i} \underline{\underline{x}}_{k}^{i}+\sum_{\substack{j=1 \\
i \neq j}}^{N} \underline{\underline{A}}_{k}^{i j} \underline{\underline{x}}_{k}^{j}+\underline{\underline{w}}_{k}^{i} \\
y_{k}^{i}=\underline{\underline{H}}_{k}^{i} \underline{\underline{x}}_{k}^{i}+v_{k}^{i}
\end{gathered}
$$

The results obtained for partially decentralized hierarchical state estimation (Quirino and Bottura, 2001) can be applied by duality to the overlapping subsystems (9) and (10). The filters are designed as follows:

Consider the approximate equation for the expanded subsystem $\underline{\underline{S}}_{i}$ :

$$
\begin{gathered}
\underline{S}_{i}: \underline{x}_{k+1}^{i}=\underline{\underline{A}}_{k}^{i} \underline{\underline{x}}_{k}^{i}+\sum_{\substack{j=1 \\
i \neq j}}^{N} \underline{\underline{A}}_{k}^{i j} \underline{\underline{x}}_{k}^{j}+\underline{w}_{k}^{i} \\
y_{k}^{i}=\underline{H}_{k}^{i} \underline{x}_{k}^{i}+v_{k}^{i}
\end{gathered}
$$

where

$$
\begin{aligned}
& \underline{w}_{k}^{i}=\sum_{\substack{j=1 \\
i \neq j}}^{N} \underline{\underline{A}}_{k}^{i j} \chi_{k}^{j}+\underline{\underline{w}}_{k}^{i} \\
& \text { with } \quad \chi_{k}^{j}=\underline{\underline{x}}_{k}^{j}-\underline{\underline{x}}_{k / k}^{j}
\end{aligned}
$$

By using (13) as a "plausible" approximation (Quirino and Bottura, 2001) to represent a white noise, we can estimate the state $\underline{x}_{k+1}^{i}$, using a set of partially decoupled Kalman filters (Quirino and Bottura, 2001) described by the following stages:

\section{Prediction Stage}

$$
\begin{gathered}
\underline{x}_{k+1 / k}^{i}=\underline{\underline{A}}_{k}^{i} \underline{\underline{x}}_{k / k}^{i}+\sum_{\substack{j=1 \\
i \neq j}}^{N} \underline{\underline{A}}_{k}^{i j} \underline{\underline{x}}_{k / k}^{j} \\
\underline{P}_{k+1 / k}^{i}=\underline{\alpha}_{k+1 / k}^{i} \underline{\underline{A}}_{k}^{i t}+\underline{Q}_{k}^{i}
\end{gathered}
$$

where

$$
\begin{gathered}
\underline{\alpha}_{k+1 / k}^{i}=\underline{\underline{A}}_{k}^{i} \underline{P}_{k / k}^{i} \\
\underline{Q}_{k}^{i}=\underline{Q}_{k}^{i}+\underline{\alpha}_{k+1 / k}^{i j} \\
\underline{\alpha}_{k+1 / k}^{i j}=\sum_{\substack{j=1 \\
i \neq j}}^{N} \underline{A}^{i j} \underline{P}_{k / k}^{j} \underline{\underline{A}}_{k}^{i j t}
\end{gathered}
$$

$\underline{Q}_{k}^{i}$ Covariance matrix of the $\underline{w}_{k}^{i}$ approximate expanded white noise;

$\underline{P}_{k / k}^{i}, \underline{P}_{k / k}^{j}$ Covariance matrices of the $i$ th and $j$ th approximate expanded subsystems, respectively.

\section{Correction Stage}

$$
\underline{x}_{k / k}^{i}=\underline{x}_{k / k-1}^{i}+G_{k}^{i} \gamma_{k / k-1}^{i}
$$

where

$$
G_{k}^{i}=\underline{P}_{k / k-1}^{i} \underline{H}_{k}^{i t}\left(\underline{H}_{k}^{i} \underline{P}_{k / k-1}^{i} \underline{H}_{k}^{i t}+R_{k}^{i}\right)^{-1}
$$

denotes the gain matrices of the local Kalman filters and $\gamma_{k / k-1}^{i}$ is the measurement prediction error of the $i$ th approximate expanded subsystem.

The covariance matrix of $\underline{x}_{k / k}^{i}$, based on $\gamma_{k / k-1}^{i}$ can be written as:

$$
\begin{gathered}
\underline{P}_{k / k}^{i}=K_{k}^{i} \underline{P}_{k / k-1}^{i} \\
K_{k}^{i}=I-G_{k}^{i} \underline{H}_{k}^{i}
\end{gathered}
$$

Due to the approximation (13), the prediction correction based on non local observations is unnecessary (Quirino and Bottura, 2001) .

Owing to overlapping decomposition, the state vectors $\underline{x}^{i}$ and $\underline{x}^{i-1}$ share the part $\underline{x}^{i-1,2}$, i.e. , $\underline{x}^{i-1}=$ $\left[\underline{x}^{i-2,2} \underline{x}^{i-1,1} \underline{x}^{i-1,2}\right]^{t}$ and $\underline{x}^{i}=\left[\underline{x}^{i-1,2} \underline{x}^{i, 1} \underline{x}^{i, 2}\right]^{t}$. 
Table 2: Sensor fault decision table for two sensors

\begin{tabular}{|c|c|c|}
\hline \multicolumn{2}{|c|}{$z_{1,2}$} & Sensor fault decision \\
\hline \multirow{2}{*}{ negative } & $\leq \varepsilon$ & fault in $y_{1}$ \\
\cline { 2 - 3 } & $>\varepsilon$ & faults in $y_{1}$ and $y_{2}$ \\
\hline \multicolumn{2}{|c|}{ positive } & fault in $y_{2}$ \\
\hline \multicolumn{2}{|c|}{ null } & normal operation \\
\hline
\end{tabular}

Let $\left[\underline{x}_{k / k}^{i-1,2}\right]_{s s 1},\left[\underline{x}_{k / k}^{i-1,2}\right]_{s s 2}$ represent the estimated values of the state vector $\underline{x}^{i-1,2}$ from the filters of subsystems $i-1$ and $i$, respectively.

For the case of two subsystems, during normal operation of the overall system we have

$$
z_{1,2}=E\left(\left[\underline{x}_{k / k}^{1,2}\right]_{s s 1}-\left[\underline{x}_{k / k}^{1,2}\right]_{s s 2}\right)=0
$$

where $E$ is the mathematical expectance.

If one or more than one of the $N$ subsystem sensors are malfunctioning, the above condition will be violated, as shown in Table 2.

As a result, $Z_{1,2}$ becomes biased (positive or negatively) because of the discrepance between estimates of the corresponding overlapping state.

Thus, by examining $Z_{1,2}$, the faulty sensors can be localised as shown in the voting decision Table 2.

The tolerance value, $\varepsilon$, which is the magnitude of the departure from zero-mean, must be found for a specific application, depending on noise considerations and on model parameter uncertainty.

हis a constant which is usually determined by the experience of the designer. However, in failure cases which are different from those considered in obtaining the value of $\varepsilon$, further investigation is required.

It is important to observe that such investigation can lead us to incorporate the failure estimation treatment into our proposal, due to the fact that different values of $\varepsilon$ are useful in characterizing the failures.

The failure estimation problem involves the determination of the extent of failure. This could be expressed by a sensor becoming completely non-operational(and be off or have hard-over failures), or by degradation in the form of a bias or reduced accuracy. The failures may be modeled as abrupt changes in the $\mathrm{H}$ matrix or as increase in the sensor covariance.

By inspecting the validity of eqn.(23), we can detect and locate the sensor failures among the $\mathrm{N}$ subsystems.

It is important to highlight that the use of decentralized

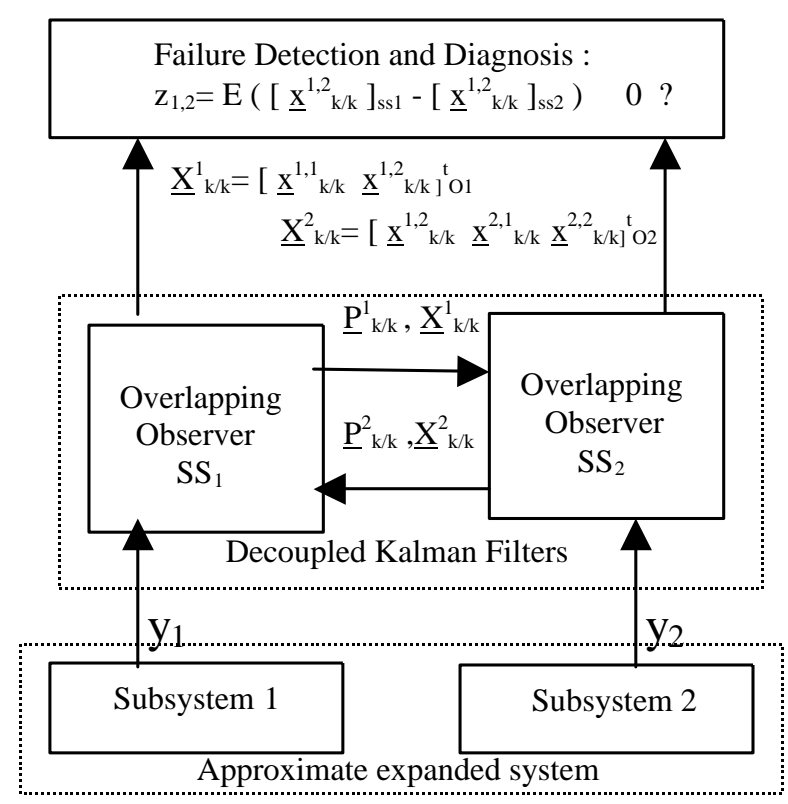

Figure 2: Approximate expanded system connected to failure detection system

estimation (14-22) modified the SFD scheme originally proposed in Hassan et al. (1992) (which uses differences between overlapping states of the subsystems), by the generation of different failure test conditions (Table 2). Another point to be noted is that in spite of not obtaining the best state estimate of $\underline{\underline{S}}$, the unbias property is preserved, meaning that the scheme above will not only be useful as a composite fault detector but also as a good state estimator (by using the inverse of similarity transformation).

Figure 2 illustrates the use of the decentralized state estimators to detect faulty sensors when subsystems $\underline{S}_{1}$ and $\underline{S}_{2}$ share the state variable $\underline{x}^{1,2}$.

Although the implementation of this SFD method requires communication between the subsystems, composite faults are precisely detected and isolated and this enhances the reliability of the SFD scheme.

From the point of view of the sensor's output, the subsystem estimators are completely decoupled, by the fact the state corrections are based on purely located observations. In other words, such state corrections don't take into account the successive orthogonalizations between the subsystems. On the other hand, these estimators take into consideration the interaction terms between the subsystems.

If the interactions between the subsystems are strong 
(i.e. strongly connected subsystems), a malfunction in any sensor could affect all the local filter estimates, and by consequence, compromise the response of the proposed SFD scheme.

Thus, it remains to show that the proposed SFD scheme also works satisfactorily in systems where the interactions may be strong, due to the fact the approximation (13) be just considered "acceptable" for weakly coupled systems (Quirino and Bottura, 2001).

In order to minimize the effect of noise, $\mathrm{z}_{1,2}$ is passed through a low-pass filter as follows:

$$
z_{1,2}^{f}(k+1)=z_{1,2}^{f}(k)+g \cdot\left[z_{1,2}(k+1)-z_{1,2}^{f}(k)\right],
$$

where $g$ is the filter gain. $g$ and $\varepsilon$ are chosen by simulations. The gain $g$ serves exclusively to smooth the estimator oscillations produced by the state and measurement noises.

In addition, if the state "Q" and measurement noise "R" covariance matrices, both diagonal, are such that the elements $q_{i i}$ are identical for all $i$ and $r_{i i}$ are identical for all $i$, then a unique gain $g$ will smooth all the state variables estimates simultaneously.

The filtered output $z_{1,2}^{f}$ is used to measure the departure of $z_{1,2}$ from zero-mean, and thus to locate the faulty sensors.

\section{APPLICATION AND SIMULATION RE- SULTS}

The results given in this section are obtained by considering a $4^{t h}$ order system with two sensors outputs. By using an appropriate transformation matrix $\mathrm{T}$ and complementary matrices $\mathrm{M}$ and $\mathrm{L}$, the approximate system is expanded into a $5^{\text {th }}$ order system consisting of two interactive overlapping subsystems.

The actual parameter values used for the original $4^{\text {th }}$ order system and the expansion obtained are given below:

\section{Original System}

$$
\begin{gathered}
x_{k+1}=A x_{k}+w_{k}+c \\
y_{k}=H_{k} x_{k}+v_{k} \\
A=\left[\begin{array}{cccc}
0,18 & 0 & 0 & 0 \\
-0,25 & 0,27 & 0 & 0 \\
0,55 & 0 & 0,18 & 0 \\
0 & 0,55 & -0,25 & 0,27
\end{array}\right] \\
c^{t}=\left[\begin{array}{llll}
4,5 & 6,15 & 2 & 2,65
\end{array}\right] \\
H=\left[\begin{array}{llll}
0 & 1 & 0 & 0 \\
0 & 0 & 0 & 1
\end{array}\right]
\end{gathered}
$$

$X_{0}=\left[\begin{array}{llll}5 & 5 & 5 & 5\end{array}\right] ; R=10^{-3} I_{2} ; P_{0}=25 I_{4} ; Q=10^{-3} I_{4}$

\section{Expanded Approximate System}

$$
\begin{gathered}
\underline{x}_{k+1}=\underline{\underline{A x}} k+\underline{w}_{k}+\underline{c} \\
y_{k}=\underline{\underline{H}} x_{k}+v_{k}
\end{gathered}
$$

$$
A=\left[\begin{array}{cc|ccc}
0,18 & 0 & 0 & 0 & 0 \\
-0,25 & 0,27 & 0 & 0 & 0 \\
\hline-0,25 & 0 & 0,27 & 0 & 0 \\
0,55 & 0 & 0 & 0,18 & 0 \\
0 & 0,55 & 0 & -0,25 & 0,27
\end{array}\right]
$$$$
c^{t}=\left[\begin{array}{ll|lll}
4,5 & 6,15 & 6,15 & 2 & 2,65
\end{array}\right]
$$

$$
\begin{gathered}
Q=10^{-3}\left[\begin{array}{ll|lll}
1 & 0 & 0 & 0 & 0 \\
0 & 1 & 1 & 0 & 0 \\
\hline 0 & 1 & 1 & 0 & 0 \\
0 & 0 & 0 & 1 & 0 \\
0 & 0 & 0 & 0 & 1
\end{array}\right] \\
H=\left[\begin{array}{ll|ll}
0 & 1 & 0 & 0 \\
\hline 0 & 0 & 0 & 1
\end{array}\right]
\end{gathered}
$$

$\underline{\underline{P}}_{0}=25 I_{5} ; R=\underline{\underline{R}}=10^{-3} I_{2}$.

The overall system is split into two interconnected subsystems, as shown by bold lines in $A$. The partially decentralized Kalman filters are calculated for the subsystems.

A system simulation, which uses the matrices $\underline{\underline{A}}, \underline{\underline{H}}, \underline{Q}$ and $\underline{\underline{R}}$ of the approximate expanded original system is used to generate the measurements $y_{1}$ and $y_{2}$.

Sensor faults are simulated as sudden changes in the appropriate elements of the measurement matrix $\underline{\underline{H}}$. The simulation results are obtained with $g=0.07$ in equation (24). In this application, $\varepsilon$ was taken to be equal to $-0,6$.

Case a: Normal operation. For this case, we have $E\left(\left[\underline{x}_{k / k}^{1,2}\right]_{s s 1}-\left[\underline{x}_{k / k}^{1,2}\right]_{s s 2}\right)=0$.

In this case, the matrix $\underline{\underline{H}}$ is kept constant so that the simulated measurements $y_{1}$ and $y_{2}$ are the sensors outputs under no fault condition. The respective estimates of the shared state are in very good agreement as can be seen from Figure 3 where the filtered difference $z_{1,2}^{f}$ is shown. This indicates that both sensors are functioning normally.

In the following cases, failures of both sensors and of one sensor at a time are assumed to occur.

Sufficient time is given for the estimators to produce satisfactory values of the estimated states before the injection of sensor failures. 


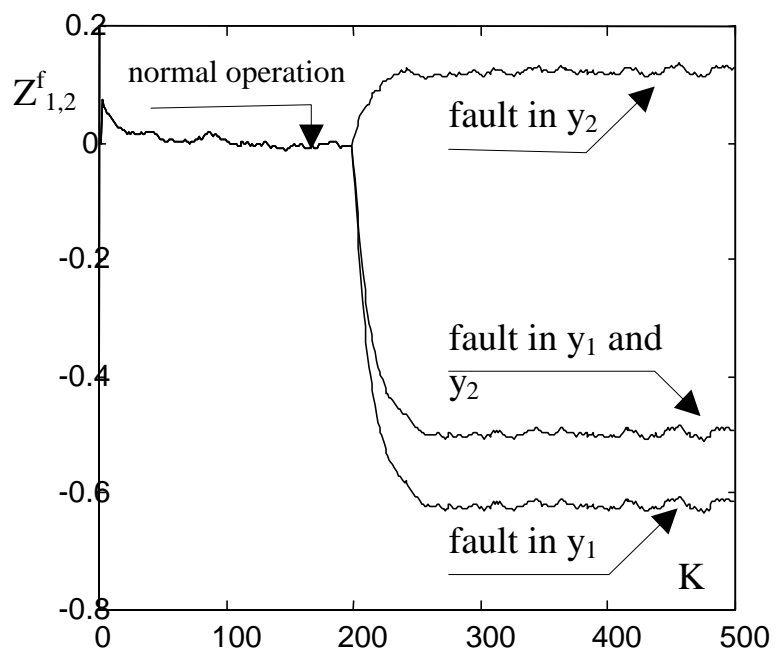

Figure 3: Cases a, b, c and d of sensor faults

Case b: The sensor of subsystem 1 failed:

$\left(\underline{\underline{H}}_{1}=\left[\begin{array}{ll}0 & 1.15\end{array}\right]\right)$ at the iteration $k=200$.

As result we have $E\left(\left[\underline{x}_{k / k}^{1,2}\right]_{s s 1}-\left[\underline{x}_{k / k}^{1,2}\right]_{s s 2}\right)<0$.

The Figure 3 shows that, after the occurrence of the fault, $z_{1,2}^{f}$ becomes negatively biased which, according to the decision scheme in Table 2, indicates a fault in sensor 1 .

Case c: The sensor of subsystem II failed:

$\left(\underline{\underline{H}}_{2}=\left[\begin{array}{lll}0 & 0 & 1.15\end{array}\right]\right)$ at the iteration $k=200$.

The filter results for this case (depicted in Figure 3) clearly indicate a positive deviation of $z_{1,2}^{f}$ from zero-mean, which corresponds to a fault in sensor 2 .

Case d: Both sensors of the subsystems failed:

$\left(\underline{\underline{H}}_{1}=\left[\begin{array}{ll}0 & 1.15\end{array}\right]\right.$ and $\left.\underline{\underline{H}}_{2}=\left[\begin{array}{lll}0 & 0 & 1.15\end{array}\right]\right)$ at the iteration $k=200$. For $\bar{\varepsilon}=-0.6$, in Table 2, these simultaneous faults are correctly detected by the filter as can be seen from the results in Figure 3.

In figure 4 , sequential failures of the sensors $y_{1}$ and $y_{2}$ are combined at the iterations $k=200$ and $k=250$. From the results in Figure 4, we can verify that the SFD scheme proposed will respond satisfactorily in diagnosing sequential failures, by the convergence of the single failure curves to that of the simultaneous failures situation shown in Figure 3 .

Since the filter calculations are performed on loworder blocks of subsystem equations, the SFD pro-

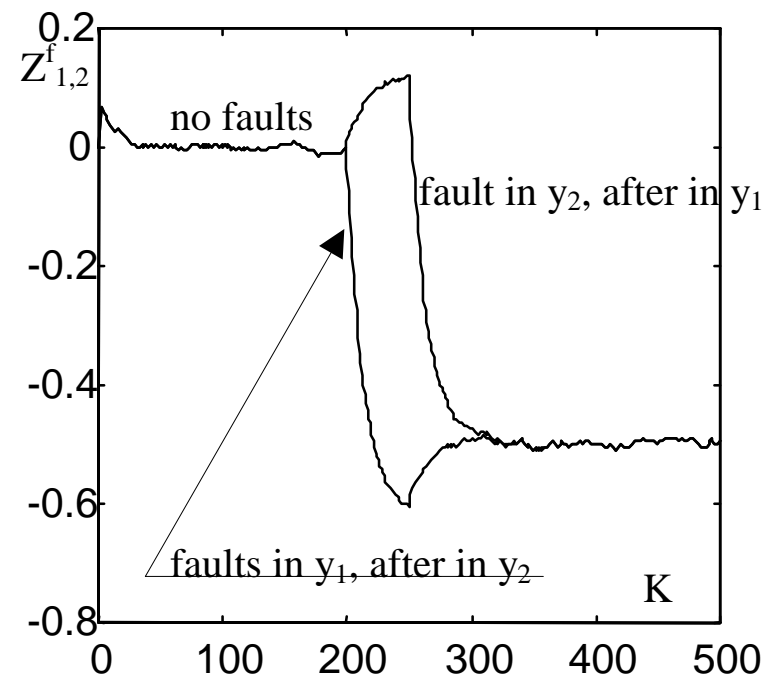

Figure 4: Sequential faults

posed can work with accuracy and numerical stability even for high-order systems.

Case e: Uncertainty in the parameters

In all the previous simulations, the partially decoupled Kalman filters were provided with the exact expansion matrices and it was assumed that the system parameters are known exactly. In practice, there may be some uncertainty about the parameter of the system and it is important to examine how this affects the SFD scheme.

To this end, the simulations performed in the cases of sequential and simultaneous faults are repeated with exactly the same conditions, except that the expanded matrix $\underline{\underline{A}}$ used in the partially decoupled Kalman filtering scheme is perturbed in order to simulate parameter uncertainty, i.e., some of the parameters have been changed by more than $10 \%$.

$$
A=\left[\begin{array}{ccccc}
0.16 & 0 & 0 & 0 & 0 \\
-0.22 & 0.19 & 0 & 0 & 0 \\
-0.25 & 0 & 0.24 & 0 & 0 \\
0.45 & 0 & 0 & 0.1 & 0 \\
0 & 0.5 & 0.56 & -0.22 & 0.27
\end{array}\right]
$$

The PDHKF (Partially Decentralized Hierarchical Kalman Filter) results obtained are given in figures 5 and 6 . It can be seen that the smoothed differences $Z_{1,2}^{f}$ have (non-zero) negative constant bias, even though there is no sensor fault.

When a fault in sensor 1 or sensor 2 or in both sensors occur, the PDHKF results are as shown in 


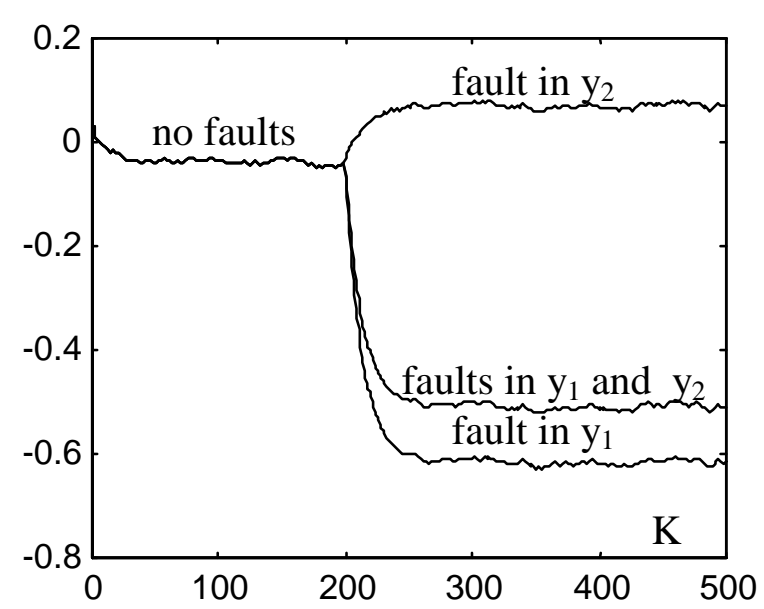

Figure 5: Single and simultaneous faults with parameter uncertainty

figures 5 and 6 . These results show a change to a different bias in $Z_{1,2}^{f}$ after the occurrence of each one of those faults.

Disturbances in the matrices $\mathrm{Q}, \mathrm{R}$, and $\mathrm{P}$ used by the PDHKF filter have also been simulated and similar results have been obtained (i.e., $Z_{1,2}^{f}$, depending on the fault locations, changes suddenly at some point in time, only when a sensor fault is present).

Although the SFD scheme can cope with small parameter uncertainty, robustness against larger uncertainty is an important consideration for practical applications and is the subject of current research.

\section{CONCLUSION}

In this paper, an extension of the Sensor Fault Detection method introduced by Hassan et al.(1992) has been proposed. The objective of the extension was to detect and isolate precisely composite sensors malfunctioning. This is achieved by using an approximation which provides estimated interactions between the subsystems as portions of system noise.

Suboptimal Kalman filters have been used to estimate the states of the overlapping subsystems and a procedure to incorporate new interactions within the filter equations has been described.

Simulation results using a low order system with two sensors have shown that the method operates satisfactorily and that discrepances between estimates of the shared states of the subsystems can be used to identify

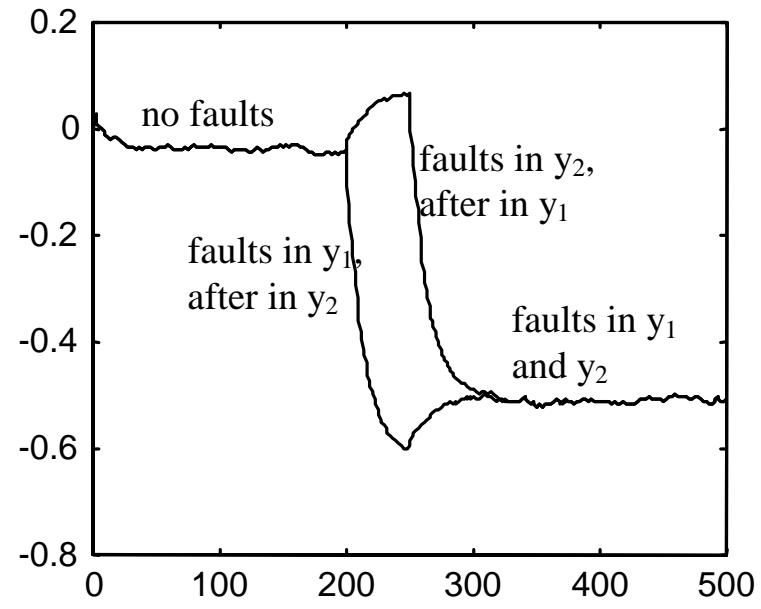

Figure 6: Single and sequential faults with parameter uncertainty

and precisely isolate malfunctioning sensors.

The method can be applied equally well to a large scale system decomposed into more than two overlapping subsystems.

\section{ACKNOWLEDGEMENTS}

The authors wish to thank the reviewers for their thoughtful and helpful comments.

\section{REFERENCES}

Benkherouf, A., and Allidina, A.Y. (1987). Sensor fault detection using overlapping decomposition . Large Scale Systems, 12, pp. 3-21.

Frank, P.M. (1990). Fault diagnosis in dynamic systems using analytical and knowledge - based redundancy - A survey. Automatica, 26 (3), pp.459-474.

Hassan, M.F., Sultan, M.A., and Attia, M.S. (1992). Fault detection in large-scale stochastic dynamic systems. IEE Proc. D, Control Theory Appl., 139 (2), pp.119-124.

Ikeda, M., and Siljak, D. D. (1980). Overlapping decompositions, expansions and contractions of dynamic systems. J. Large-Scale systems,1, pp.29-38.

Ikeda, M. , Siljak, D.D. , and White, D.E . (1981) . Decentralized control with overlapping information sets. J. Optimization, Theory and Application, 34, pp. 279-310. 
Isermann, R. (1984). Process fault detection based on modelling and estimation methods - A survey. Automatica, 20 (4), pp. 387-404.

Krtolica, R., and Siljak, D.D. (1980). Suboptimality of decentralized stochastic control and estimation. IEEE Trans. Autom. Control, AC-25, pp. 76-83.

Quirino, R.B. , Bottura, C.P., and Costa Filho, J.T. (1998). A computational structure for parallel and distributed Kalman filtering. Proceedings of the XII Congresso Brasileiro de Automática, pp. 747753.

Quirino, R.B. , and Bottura, C.P. (2001).An approach for distributed Kalman filtering. Revista Controle 83 Automação da SBA (Sociedade Brasileira de Automática), Vol.12, No.1.

Singh, M.G. , Hassan, M.F., Chen, Y.L., and Pan, O.R. (1983). New approach to failure detection in largescale systems . IEE Proc.D, Control Theory Appl., 130 (5), pp. 243-249.

Willsky, A. (1976). A survey of design methods for failure detection in dynamic systems. Automatica 12, pp.601-611. 